\title{
Traduction Et Bilinguisme: Traduire L'altérité À L'heure Du Bilinguisme Et Du Multiculturalisme Au Cameroun
}

\author{
Jean-Richard DONGHO* \\ École Supérieure de Traducteurs et Interprètes (ASTI), Université de Buea, Cameroun
}

*Corresponding Author: Jean-Richard DONGHO, École Supérieure de Traducteurs et Interprètes (ASTI), Université de Buea, Cameroun

\begin{abstract}
This reflection is articulated around translation as a contribution towards togetherness in Cameroon. Translation is understood here as a doublecarriageway linking the two sociolinguistic spaces of Cameroon. This reflection-demonstration focuses on the import/export of Anglophone culture and identity into the French-speaking world. It is based on the assumption that translating official texts as well as literary works simply by seeking genius of the language or fluidity of expression is a dangerous naivety. In view of the ambient otherness, in a context of identity crises, the result would be counterproductive, in that the translations would systematically mask the cultures and identities of the source language/culture while enhancing the visibility of the reception context. The article illustrates the urgent need for this ethical shift by studying the translatability into French of literary texts not yet translated from the English-speaking region. It emerges from these pre-translational explorations that this metonymy of contemporary English-speaking literature conveys an English-speaking culture that is anything but monolithic. It constitutes a space of contact and openness which invites and welcomes all the languages/cultures of the Cameroonian space, including French. The ethics of translating would consist in the importation of the plural Anglophone identity by decentering and foreignizing Anglophone culturemes. This way of translating, which should be transposable to the French - English translation, respects and integrates into the translations the cultures, identities and world views of both the source and target languages. This culturally inclusive translation could well serve as silt in the construction of a typically Cameroonian goût.
\end{abstract}

Keywords: Otherness, bilingualism and multiculturalism, ethics of translation, English literature

\section{Mise En PeRSPective}

En tant qu'intervention, le bilinguisme (synonyme de multilinguisme) peut s'entendre comme l'ensemble des mécanismes sociaux et institutionnels visant à instituer, restaurer ou renforcer la cohésion sociale, l'intégration ou le vivre-ensemble entre les différentes langues en contact au sein d'un espace politique donné. La question linguistique (Bamgbose, 1991) semble consubstantielle à l'entité politique Cameroun.Face à une unité nationale lézardée par les tensions successives qui ont culminé dans la crise dite anglophone, la traduction, fille de la politique nationale du bilinguisme, devrait pouvoir, à moyen et à long termes, jouer son rôle de médiation dans la recherche du vivreensemble. Paradoxalement, Le Cameroun,terre de traduction depuis l'époque coloniale (Nama, 1990 ; Mopoho, 2001),ne semble pas disposé à tirer parti du potentiel de la traduction dans la recherche de l'idéal politique de l'intégration nationale. D'une part, les textes officiels et autres textes d'utilité publique continuent soit de se publier en une langue, soit de souffrirde traductions pour le moins approximatives. D'autre part, àl'instar de la littérature africaine en général, la littérature camerounaise dans sa globalité semble se méfier ou redouter la traduction; et dans cette marginalité traductionnelle,la littérature anglophone camerounaise est la moins traduite des deux espaces culturels du pays. Entre 2002 et 2011, environ $14 \%$ des œuvres camerounaises étaient traduites et $90 \%$ desdites œuvres appartenaient à la littérature camerounaise francophone(Meli, 2011 :5).Ce constat traduit une réserve certaine de deux sous-cultures censéesaller à la rencontre de l'une et de l'autre dans un dialogue interculturel camerouno-camerounais. Comment comprendre cette attitude peu favorable à la traduction de la part du monde anglophone camerounais ? 


\section{Le Ver Dans Le Fruitdu Bilinguisme Et Du Vivre-Ensemble Au Cameroun}

Une exploration diachronique et synchronique des contacts entre l'anglais et le français dans l'espace politique camerounais montre que la méfiance de l'espace anglophone envers la traduction peut s'attribuer à nombre de facteurs dont le différentiel de pouvoir entre les communautés linguistiques officielles et le contraste entre la visibilité de l'anglais en contexte camerounais dans la géopolitique internationale en cette ère de mondialisation; et laperception de la traduction et des traducteurs dans le champ social en général, et plus particulièrement, les implicatures idéologiques sous-jacentesà la traduction domaine français.

À la faveur de ces différents temps qui ont marqué l'Histoire du monde contemporain, le Cameroun a accueilli sur ses côtes et sur son sol les représentants des différents pays européens du Portugal à la Couronne britannique et la France en passant par l'Espagne et l'Allemagne. Les exigences de la communication officielle et des contacts internationaux, la difficulté de choisir parmi la pléthore des langues locales, et l'impérieux besoin de consolider la fraternité retrouvée entre les deux sous composantes orientent le pays vers le maintien de l'anglais et du français comme langues officielles. Cettepolitique de bilinguisme masquait mal au moins deux réalités : le sentiment d'insécurité qui caractérise tout individu, toute communauté ou entité minoritaire partageant son espace avec une autre majoritaire. De plus, les Southern Cameroons qui retrouvent leurs frères de la République imaginaient-ils seulement combien ils étaient devenus différents en termes de vision du monde ?

En effet, la distance culturelle consécutive à un demi-siècle de séparation sous des schémas politicoadministratifs non seulement différents mais diamétralement opposés semble avoir échappé à des générations des décideurs politiques depuis les indépendances du Cameroun. Une sociolinguistique de la suspicion s'est ipso facto développée et s'est complexifiée au fil du temps avec la compréhension différenciée qu'ont les deux communautés de la notion d'égalité, fondement de l'union (Nkwi, 2004 :186).Néanmoins, les anciens sujets de la Couronne britannique ont adopté sans sourciller et sans contrepartie la plupart des attributs de la partie francophone : le code de la route, la monnaie, le système de gestion et d'administration. Au fil du temps, en plus d'une répartition des postes gouvernementaux jugée trop favorable à la partie francophone(Akere, 1991),ce même peuple va avoir le sentiment de vivre dans un ghetto où les contraignent leurs congénères francophones qui, plus grave encore, auraient hérité de l'ancien colonisateur un système autoritaire et centralisateur (Kom, $2000: 23-4)$. La question anglophone empoisonne les relations entre les Anglophones et les Francophones mais surtout entre la communauté anglophone et le pouvoir central. Pour lutter contre ce qu'elle perçoit comme la francisation de sa culture, la communauté anglophone s'arcboute et défend avec la dernière énergie le système éducatif et le système juridique hérités de l'ancienne métropole.

D'un point des contacts de langues et des relations internationales, le pouvoir de la minorité anglophone n'est pas exclusivement endogène. Il tient dans une large mesure à l'appartenance à la communauté linguistique anglophone internationale aux côtés des grandes puissances régionales et planétaires - Etats-Unis, Canada, Royaume-Uni, Afrique du Sud, Nigeria. D'autre part, la volte-face intervenue au Cameroun dans la loyauté envers les langues officielles depuis les années 1990 semble avoir rappelé à la communauté anglophone sa dignité à voix basse. C'est sans doute la lecture qu'elle s'est donnée de la ruée à sens unique vers l'anglais et vers le système éducatif anglophone par les Francophones depuis l'admission du Cameroun au Commonwealth (voir Anchimbe, 2011 ; Dongho, 2006) ${ }^{1}$. Comme les Grecs de l'antiquité, et les Américains et les Britanniques d'aujourd'hui, l'attrait de l'anglais a fini par développer chez cette minorité un sentiment confus : un complexe de supériorité mais, en même temps, une peur morbide de l'envahissement - une sorte de francophobie ${ }^{2}$.

Nombre d'autres facteursont plus à voir avec l'invisibilité de la traduction et du traducteur qu'avec l'histoire politique du Cameroun. Ne serait-il pas incongru, en effet, d'espérer voir la traduction logée

\footnotetext{
${ }^{1}$ Après la Réunification, ce sont les Anglophones qui se ruaient vers le système francophone en quête de mobilité verticale

${ }^{2}$ Sentiment si profond qu'il s'est créé une onzième province imaginaire où se rejettent les Anglophones non originaires des deux régions anglophones
} 
à meilleure enseigne que le traducteur dans cette société mondialisée? De manière générale, en effet, cet indispensable passeur culturel occupe un champ social plutôt périphérique: souvent comparé ou confondu à la machine, le traducteur est considéré comme un simple canal ou un simple relai de la pensée d'autrui. De plus, une des sentences - malheureusement - les plus connues sur la traduction reste Traduttore, traditore ${ }^{3}$. Cet axiome, qui est un des lieux-communs les plus tenaces sur la traduction, est tout à fait significatif de l'estime en laquelle on la tient (Liévois, 2018 :3-4). La traduction induirait plus la méfiance que la confiance, plus la suspicion que l'assurance, plus la fermeture et l'éloignement que l'ouverture et le rapprochement. Un autre argument sous-jacent au refus de l'emploi de traductions reste celui de l'infériorité de la version traduite par rapport au texte original ou texte-source - C'est généralement l'original qui fait foi. On tient l'analyse de traductions pour moins intéressante que celle d'originaux.

De plus, les enjeux de la traduction dépassent l'opération technique de transposition linguistique. Loin des fonctions didactiques et professionnelles, elle est souvent le lieu de manipulations lourdes de conséquences sur les plans politique et idéologique. En effet, la manière de traduire - les stratégies et les procédés- utilisée par les traducteurs a une incidence réelle sur le traitement de la culture en traduction : autant les approches ciblistes rehaussent la culture d'arrivée passantpar la culture source sous l'éteignoir ; autant les approches sourcistes font la part belle aux valeurs culturelles sources dans le produit de l'opération au détriment de la culture d'arrivée. La tradition française de la traduction se caractérise par la quête d'idiomaticité. Dans cette tradition, les traducteurs recherchent en priorité la clarté, l'élégance, et le rythme. Pour ce faire, ils en viennent, à moderniser, retrancher, ajouter, se préoccupant des rapports logiques et des transitions bref à critiquer et à corriger les auteurs qu'ils traduisent. Comme Amyot au XVIe siècle, ils pratiquent la «francisation»: ils suppriment ou atténuent les passages qui choqueraient les beaux esprits de leur temps. Des traductions qui,pour plaire et se conformer au goût de leur époque, sont des versions «revues et corrigées » par des traducteurs conscients (trop sans doute) de la supériorité de leur langue et de leur jugement (Horguelin, $1980:$ 76).

Cette manière de traduire apparentée aux «Belles Infidèles ${ }^{3}$ du classicisme français se reproduit discrètement de nos jours dans le domaine français, surtout dans sa célèbre théorie interprétative du sens (Seleskovitch et Lederer, 2014). Comme lemartèle Berman ( 1984: 53-4) «Poser que le but de la traduction est la captation du sens, c'est détacher celui-ci de sa lettre, de son corps mortel, de sa gangue terrestre. C'est saisir l'universel et laisser le particulier. » Pourtant, c'est le travail sur le corps et la lettre du texte qui singularise le texte littéraire et, normalement, sa traduction. Sur ces entrefaites, la méfiance de la communauté anglophone envers la traduction pourrait se justifier par la conscience du péril que constitueraient des Belles Infidèles pour leur identité menacée de francisation.

La conjugaison de ces facteurs a contribué, sans doute, à l'invisibilité de la traduction dans l'espace politique et littéraire anglophone. Pourtant, la traduction demeure le lieu de présentation et de réception de soi et de l'autre à d'autres champs culturels; et c'est grâce à leur dissémination par les traductions que les littératuresémancipent les peuples en jouant leur rôle demiroir culturel.

\section{TRAdUCTION COMME INTERVENTION}

Les textes officiels, politiques, et culturels ont ceci de commun qu'ils touchent àl'âme d'un peuple, sa vision du monde, des valeurs mais aussi des aspirations, des appréhensions et autres rancœurs accumulées dans le subconscient collectif d'une communauté au cours de son Histoire. Ces composantes de l'identité culturelle constituent la marque distinctive de chaque communauté. Comment donc connaitre un peuple sans accéder à ces informations ? ${ }^{4}$ Au-delà des autres caractéristiques et fonctions sociales, le dialogue interculturel qui s'opère par le biais de la traduction littéraire sert d'antidote contre ce poison social que sont les préjugés et les stéréotypes. Ndeffo (2015) fait état de deux projets menés en Allemagne permettant de mesurer l'importance de la traduction dans la promotion du dialogue entre les cultures et la gestion des conflits dus à la méconnaissance de l'Autre. Deux institutions ont décidé de combattre la méconnaissance des cultures étrangères en

et ses variantes françaises : «Traduire, c'est trahir », «traduction - trahison » ou encore «traducteurs, vous êtes des traitres »

${ }^{4}$ Commel'affirme Thiele (1967 :2) "Knowledge of a country, not only of the present, but of the past is a bridge to understanding, and understanding is a key to friendship" 
Allemagne par le biais de la traduction littéraire. C'est ainsi que, pour faire tomber les préjugés entre la population allemande et les pays du reste du monde, les littératures des pays d'Afrique, d'Amérique latine et d'Asie et du monde arabe ont été traduites et publiées en Allemagne pour les lecteurs germanophones. On pourrait penser que le Cameroun, qui entretient d'ailleurs une relation historique avec l'Allemagne, s'inspirerait de ces projets dans le cadre de sa politique nationale du bilinguisme et du multiculturalisme, mais plus spécialement dans la recherche du vivre ensemble.

D'un point de vue officiel, le Cameroun est composé de deux univers géographiquement intégrés mais socialement et culturellement étrangers, distants sans possibilité de franchissement de cette ligne Maginot sur les bords de laquelle campent les deux culturesqui se regardent en chiens de faïence. Comme le relève AtebaNgoa (2004:71) et Ndeffo (2015:267), le Cameroun abrite deux littératures qui se côtoient sans communiquer : ces deux littératures reflètent les deux communautés qui vivent ensemble sans vraiment se connaitre, et subissent un conflit plus ou moins ouvert.

Rien n'empêcherait la communauté anglophone de se reconnaitre dans Banda, si Ville cruelle d'Eza Boto leur était présentée. Les tribulations et déboires du jeune paysan sont en effet loin d'être l'apanage d'une seule communauté du pays. Ceux des Anglophones qui pensent être victimes d'une conspiration et d'une marginalisation orchestrées par les Francophones constateront que leur sort est partagé par leurs compatriotes francophones depuis l'époque coloniale (Ndeffo, 2015 :267).

Dans le cas de la traduction en français des textes en anglais, l'auteur relève,

Ce n'est qu'en prenant conscience de ces malheurs racontés par les victimes elles-mêmes que les lecteurs francophones pourront comprendre les frustrations de la communauté anglophone et prendre conscience du rôle qu'ils jouent (ou ont joué) consciemment ou non, pour provoquer ou entretenir la misère de celles-ci (Ndeffo, 2015 :268).

Les analyses et les propositions de ces auteurs sont d'une pertinence certaine. Cependant, elles semblent perdre de vue la manipulation inhérenteà l'acte de traduire, et la nécessité de bien choisir la manière de 'bien' traduire'. En effet, l'acte de traduire est essentiellement intervention; et l'insouciance théorique et méthodologique et stratégique destraducteurs transforme ces derniers, de facto, en agents d'annexion des textes étrangers. En dépit du discours sur la pratique réflexive, la fracture entre l'art de traduire et la réflexion sur cet art est généralement profonde (Wagner et Chesterman, 2002). Comme semble le relever pour le regretter Venuti (1992:1-4-6), les traducteurs contemporains sont arcboutés sur la production ou l'esthétique se sevrant de toute réflexion méthodologique et stratégiqueserrée sur leur art, leur métier et leur profession. Du coup, les traductions se livrent sans commentaires ni critique encore moins de précisions relatives aux conditions de production,aux structures institutionnelles qui déterminent la consommation, lacirculation et la réception de textes traduits ${ }^{5}$.

Dans la pratique, certains modèles fonctionnels ont érigé, de manière subliminale, en sacro-saint principe, le fait que la traduction ne doit pas sentir la traduction. Par conséquent, les seuls critères de qualité souvent attendus et exigés des traductions littéraires demeurent la lisibilité et la fluidité du texte. Consciemment ou pas, se plier à cette double exigence des forces du marché revient à soumettre le texte traduit au diktat exclusif du contexte et de la culture cibles : à le domestiquer ! D'un point de vue historique, la traduction domaine français se singularise par la recherche du sens et de l'idiomaticité.

L'anguille sous la roche de l'approche interprétative reste la perception de la traduction comme conquête ou soumission de l'Autre, Comme le poétise Bourjea (1986:231-2) ce traducteurconquistador culturel emporte dans ses bagages tout un arsenal de valeurs esthétiques et morales à imposer au lectorat cible: Sans en donner l'air, le traducteur-conquistador joue le jeu de l'occupant/occupation: «Usant d'un appareil linguistique, il soumet et convertit, il s'approprie. À coup de violence et de séduction, il acclimate » (Bourjea, 1986 :232). Pourtant, tout processus traductif est le résultat d'un ensemble d'influences, de tensions et de contraintes culturelles,

\footnotetext{
${ }^{5}$ « They appear as esthetically sensitive amateurs or talented craftsmen, but not critically self-conscious writers who develop an acute awareness of the cultural and social conditions of their work. Venuti (1992 :1-4-6) »
} 
éditoriales et politiques géo-historiquement déterminées; il rend visibles les rapports de forces qui s'établissent entre les «lieux» de la traduction, dans le cadre plus large des dynamiques de circulation des textes, des langues et des imaginaires. $»^{6}$. Au Cameroun comme dans tous les pays multilingues et multiculturels, les lieux de la traduction demeurent des instances de négociations des différences linguistiques, socioculturelles et identitaires.

Les contacts de cultures dans contexte international de la mondialisation sontmarqués par la résurgence et l'accentuation des replis identitaires. Par conséquent, chaque peuple a tendance à phagocyter ses halogènes et à leur dicter une vision du monde, un mode d'exister et une langue, qu'ils ne comprennent que peu ou pas du tout (Kamguem 2021 :25). Ramenéeà l'échelle nationale, ce dépaysementà outrance de l'Autre pourrait se décline, en terme de stratégies de traduction, en 'Sawanisation, Anglophonisation, Graffinisation, Baminisation, Come-nogonisation, ou Francophonisation' des traductions. C'est cette stratégiecannibalisante des langues coloniales qui a actuellement pignon sur rue dans les anciennes colonies. En effet, nombre d'États revendiquent une identité spécifique et s'attachent à authentifier leur spécificité culturelle en se détachant de la langue coloniale et en forgeant leur propre identité linguistique dans une créolisation anti-hégémonique féconde et séditieuse (Boufoy-Bastick, 2012 : 16).

Le Cameroun fait bien partie de ces contextes postcoloniaux. Cependant, la nation depuis les indépendances des deux Camerouns se bat pour maintenir ensemble non sa multitude ethnolinguistique proche du babélisme, mais les deux composantes sociolinguistiques consécutives mais également consubstantielles au passage à la souveraineté internationale. La politique nationale du bilinguisme officiel se décline dans toutes les situations de contacts entre les deux sous espaces majeurs de la nation. Malgré toutes les précautions, la cohabitation a, au fil du temps, amené la minorité anglophone àredouter et às'opposer farouchement à la francisation de la culture et de l'identité anglophones. Par conséquent, si d'une part traduire en hégémonisant une sous composante ethnique reste sans objet dans la recherche du vivre-ensemble, d'autre part, coller à la traduction ethnocentrique en français des textes littéraires de ce terroirconstituerait une grave erreur : les textes seront trop accommodants pour la langue française et la culture francophone en passant sous l'éteignoir les valeurs, les représentations et les idéaux de la communauté anglophones.Considérant la recherche de l'équité qui sous-tend la politique du bilinguisme officiel, une telle acclimatation filtrante des textes serait perçue comme un viol ou une provocation tant elle est violence, injustice voire trahison!Ce qui risque d'être perdu par la même occasion ici c'est justement ce qui fait de l'interculturel un espace d'instabilité, d'hétérogénéisation, de remise en question et de réinvention des relations sociales, un espace où la différence n'est pas effacée par un transfert technique mais, au contraire, intervient sur la relation sociale pour la transformer, un espace d'altération justement où tous les interlocuteurs sont traduits y compris le traducteur (Chaouite, 2008 :18). Et au traducteur de comprendre avec Wuilmart (2007) que :

Sa fidélité doit être double, et concernera non seulement la culture et la langue réceptrices, mais aussi, et même surtout, celles qu'il a pour vocation de transposer. [...] Et le jeu en vaut bien la chandelle, puisque cette attitude profondément altruiste et tolérante, consistant à transformer son propre terrain linguistique en terre d'accueil véritable, fera de ce traducteur de bon aloi le plus authentique des ambassadeurs culturels.

Au vu de ce qui précède, face à une communauté anglophone camerounaise en proie à une insécurité linguistique permanente, le traducteur des œuvres littéraires anglophones du Cameroun doit, puisqu'il en a la possibilité,travailler à effacer les distances tout en sauvegardant les différences dans un esprit d'amoureuse complicité; et la traduction un dialogue libre et responsable avec l'Autre, un déplacement du texte source dans une langue qui ne se conforme plus strictement à la langue d'arrivée, mais qui n'est pas sans affinité avec la langue de départ (Bourjea, 1986 :232).Dans les lignes qui suivent, l'hypothèse d'un traduire pacifiant, libéré de toute visée impérialiste, va s'illustrer à travers une étude en traduisibilitéd' un corpus d'exemples relevant du terroir anglophone camerounais.

\footnotetext{
${ }^{6}$ Appel à contributions, Colloque international: "Space in translation. Géographies de la traduction / espaces de traduction" (Modena), 9/10 mai 2019. Voir aussi (Hersent, $2007: 105) »$.
} 


\section{Traduire La Specificite Culture Anglophone}

Trempés dans la postcolonie, les textes de la littérature anglophone brillent par une écriture de la marginalité. Cetteesthétique est le parfait corollaire de la résistance (Ngoubolong, 2020) de la minorité face à l'effacement redoutée de l'identité anglophone au contact de la majorité francophone.

Pour des besoins de démonstration, les extraits viennent des textes non encore traduitset porte sur les éléments culturellement spécifiques à la culture anglophone. Il s'agit des éléments textuels à fort contenu culturel, sans équivalents directs en langue française. Ces saillies prennent la forme de choix de mots et de constructions grammaticales récurrentes, d'un texte a l'autre mais également d'un autre à l'autre. Conformément au dépaysement qui se dégage de ces textes dans sa globalité, ces textes fourmillent d'éléments en langues étrangères : le français, les langues locales des zones anglophones comme le bafut, lemenemo, les langues locales de la partie francophone telles que le basaa, l'ewondo et le fufulde, mais aussi les langues véhiculaires que sont le francamglais etle CameroonPidgin English. De ce point de vue, cette écriture est plutôt inclusive, contrairement à une attendue polarité anglais-français. Ces éléments étrangers sont introduits sans explication ni traduction et sans stratégie apparente de reprise. Dans ce métissage linguistique et culturel, les textes ne comprennent ni notes de bas de pages ni glossaire en appendice. C'est ce tissu linguistique ouvert, voire dérangeant qui constitue la particularité des textes de la littérature camerounaise contemporaine en général, et qui élèveau carré l'altérité du texte (Denti, 2017) et le dépaysement du lecteur déjà dans sa langue du terroir.

La présente démonstration est une exploration pré-traductionnelle qui emprunte aux déclinaisons méthodologiques de la théorie des poly systèmes (Lambert et Gorp, 1985) dans une approche systématique et raisonnéeempruntéea l'esprit de la critique des traductions (Berman, 1995).

D'un point de vue pratique, tout traducteur conscient du contexte et des enjeux de son travail devra s'arrêter un instant sur comment traduire cette mosaïque, une langue si écorchée vive, sans trahir le dérangement voulu par l'auteur du texte source ? Conformémentà la dynamique relevée plus haut, il importerait de traduire dans un français qui ouvre son espace à la culture anglophone exprimée du texte source.Ce n'est surtout pas en remplaçant ces éléments par des équivalents en français standard et en les moulant simplement dans le texte en français. Pour mieux ressortir l'étrangéité du texte de départ, la traduction des xénismes pourraient paraitre en italiques dans le texte suivi des notes de bas de page (N.D.T). Toutefois, étant donné le nombre élevé de xénismes de certains textes (ex. ImmortalSeed) et l'encombrement que les N.D.T entraineraient dans le corps du texte, les traductions pourraient paraitre en fin de volume dans un glossaire de termes étrangers (cf. Sardin, 2007 : parag.24). D'ailleurs, certains écrivains africains anglophones (d'Achebe à Alex La Guma) n'hésitent pas à joindre des notes explicatives en fin de volume pour éclairer leurs lecteurs lorsqu'ils ont introduit dans leur texte des notions propres à leur culture. [...] (Sevry, 1998 :parag28).

Exemple1. Mbéré et mbéré-kaki (ImmortalSeed de TahProtus)

\begin{tabular}{|c|c|c|}
\hline & ГC1: & TC2 \\
\hline $\begin{array}{l}\text { I remember when I was a } \\
\text { mbere, } \\
\text { I remember when I treated } \\
\text { drivers, } \\
\text { I remember when I filled my } \\
\text { pockets, } \\
\text { I remember when I was } \\
\text { ambere... } \\
(\text { ImmortalSeedp.5) }\end{array}$ & $\begin{array}{l}\text { Je me souviens quand j'étais agent de } \\
\text { police, } \\
\text { Je me souviens quand j'abusais des } \\
\text { chauffeurs, } \\
\text { Je me souviens quand je me } \\
\text { remplissais les poches, } \\
\text { Je me souviens quand j'étais agent de } \\
\text { police... }\end{array}$ & $\begin{array}{l}\text { Je me souviens quand j'étais } \\
\text { mbéré, } \\
\text { Je me souviens quand j'abusais } \\
\text { des chauffeurs, } \\
\text { Je me souviens quand je me } \\
\text { remplissais les poches, } \\
\text { Je me souviens quand j'étais } \\
\text { mbéré... }\end{array}$ \\
\hline
\end{tabular}

\section{TS :}

Come and see ambere-khaki!he said with a naive smile to his elder sister. (ImmortalSeedp. 22)
TC1:

D'un air naif et avec un sourire, il dit à sa sœur ainée: «viens voir un agent de police ».

\section{TC2:}

D'un air naïf et avec un sourire, il dit à sa sœur ainée : « viens voir unmbéré-kaki. »*.

Entrée du glossaire pour mbéré et mbéré-kaki 
Mbéré: n.m. (Prononciation accentuée sur la première syllabe) : le mbéréest un terme générique à connotation péjorative utilisé dans le parler jeune des grandes villes - le francamglaiset camfranglais pour désigner les agents des corps de sécurité et de défense. Le mbéré-kakiou mange-mille, qui constitueune sous-catégorie de ce corps social, renvoie aux fonctionnaires de police dont l'uniforme était de couleur kaki jusqu'aux années 1990. Soldat, gendarme ou agent de police, le mbéréest associé aux dérives comportementales en tous genres : abus de pouvoir, répression brutale, corruption, promiscuité sexuelle, etc.

Exemple2. Across the Mongolo, Nkemngong N.

\begin{tabular}{|l|l|l|}
\hline TS : & TC1: & TC2: \\
\hline Datgendarmes & Ces gendarmes & Céré \\
dem be tifpipi I & sont des plus & sont des \\
show'am book, & véreux. Je présente mes & bandits. Je leur \\
book correct, e say & pièces, elles en ordre, mais ils & présente mes papiers. \\
a don run motuar & prétextent que & Ils sont à jour. Ils \\
too much. (Across the Mongolo, & j'étais en excès de & me disent que je faisais de la \\
p.39) & vitesse. & vitesse. \\
\hline
\end{tabular}

Pour Kamguem (2020:79) Le concept de «gendarme» du texte source n'aura pas besoin de traduction à proprement parler dans la mesure où il est déjà dans une langue parfaitement compréhensible chez l'anglophone Camerounais. Dans un esprit de bienveillante inclusivité, la traduction en français pourra simplement tendre la main à l'anglais (TC2).

Exemple3. A Widow's Might, Ngemngong $N$.

\begin{tabular}{|c|c|c|}
\hline TS: & TC1: & TC2: \\
\hline $\begin{array}{l}\text { People attributed it to some } \\
\text { mysterious cause, witchcraft, } \\
\text { poison, or ngrimba. Some } \\
\text { people were of the opinion that } \\
\text { honorable Mbutuku used } \\
\text { mysterious means to grow in } \\
\text { politics. (p. 15) }\end{array}$ & 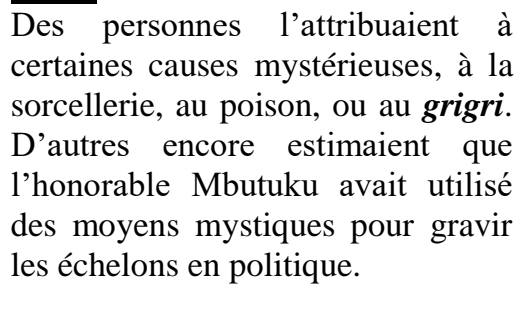 & $\begin{array}{l}\text { Des personnes l'attribuaient à } \\
\text { certaines causes mystérieuses, à la } \\
\text { sorcellerie, au poison, ou au } \\
\text { ngrimba. D'autres encore } \\
\text { estimaient que l'honorable } \\
\text { Mbutuku avait utilisé des moyens } \\
\text { mystiques pour gravir les échelons } \\
\text { en politique. }\end{array}$ \\
\hline
\end{tabular}

Après l'annonce du décès de l'honorable, la nouvelle s'est répandue comme une trainée de poudre dans toute la ville. Et comme en Afrique la mort naturelle n'existe presque pas dans les esprits, les langues se sont déliées et plusieurs attribuaient la disparition de l'homme politique aux pratiques occultes, une solution fort courue dans cette communauté également, surtout en situation de concurrence et rivalité dans divers domaines et situations de vie dont l'amour et le pouvoir politique. Le mot ngrimba est synonyme de sorcellerie, de fétichisme, ou gri-gri. Originellement du parler jeune des régions de l'ouest du Cameroun, ce terme du camfranglais est désormais d'un usage plus répandu y compris chez les adultes. La possibilité de traduction TC1 dénaturalise le texte à travers la dilution de cet arome originel alors que le piquant du texte semble refaire surface dans une traduction dépaysante, certes, mais qui conserve le parfum de la culture Grassfields aux côtés de la lettre de la langue française (TC2).

Comme le montre l'analyse des extraits ci-dessus, pour un texte à la microstructure saillante de xénismes et d'images, la traduction interprétative du sens autoriserait le traducteur à raboter les aspérités porteuses de la beauté de la culture anglophone. Se comporter ainsi rappelle cet étudiant qui prétend être excellent traducteur du seul fait que ses traductions sont rendues dans un français impeccable (Wuilmart, 2007). En plus de perdre cette diversité accommodante et ce qui fait l'unicité de la corporéité du texte de Tah, cette déverbalisation déboucherait sur une altération de la couleur du texte, àun texte informatif dénué de toute dépression stylistique, aux antipodes d'un texte littéraire. Soustraire le lecteur francophone à l'artillerie verbale du texte anglais, c'est l'installer dans une zone de confort cognitif que l'auteur n'a pas souhaité établir : c'est trahir !

\section{Pour Aller Plus loin}

La présente démonstration concerne et informe les différentes parties prenantes de la géographie de la traduction au Cameroun. Pour les traducteurs comme pour les éditeurs et autres mécènes culturels, 
elle attire l'attention sur les enjeux socio-politiques et idéologiques de la traduction officielle au Cameroun. Elle constitue un moment de prise de conscience de la responsabilité et dans le dialogue des cultures dans le vaste chantier de construction nationale.Les différents instruments relatifs au bilinguisme institutionnel au Cameroun insistent sur la signature, la publication et la circulation des textes officiels dans les deux langues officielles. Cette recherche de l'équité dans la communication officielle accordent de jureun rôle central à la traduction dans le sillage du bilinguisme. Pourtant, l'absence de traduction figure encore en bonne place des revendications catalytiques de la crise dite anglophone qui a éclaté en 2016. C'est reconnaitre désormais que le véritable enjeu réside moins dans l'idée de livrer les textes influençant la vie de toute la nation dans une seule langue que dans la 'manière de traduire' ces textes. Par conséquent, la manière de traduire les textesdevraient faire l'objet del'encadrement de la législation au même titre que les autres aspects de la vie nationale.Ainsi légiférer sur la manière de bien traduire constituera, à coup sûr, une avancéeperceptible vers la formation d'un goût singulièrement camerounais dans ses langues officielles.

\section{REFERENCES BIBLIOGRAPHIQUES}

[1] Abessolo, P.M. (2015). L'enseignement du français en zone Anglophone au Cameroun. Sous la direction de Martial Abessolo. Kansas City : Miraclaire Academic Publications.

[2] Anchimbe, E.A. (2011). Socio-historical Landmarks in Francophone Resistance to English. Revue Internationale des Arts, Lettres et Sciences Sociales (RIALSS), 1(4)1-21.

[3] Atangana Nama, C. (1990). A History of Translation and Interpretation in Cameroon from Precolonial Times to Present. Meta,35 (2), 356-369.https://doi.org/10.7202/003694a

[4] Ateba, N.M. (2004). La littérature camerounaise et les enjeux de la traduction. VoundaEtoa, M. (éd.) La littérature camerounaise depuis l'époque coloniale: Figures, esthétiques et thématiques. Yaoundé : Presses Universitaires de Yaounde.71-78.

[5] Bamgbose, A. (1991). Language and the Nation: the Language Question in sub-Saharan Africa. Edinburgh: Edinburgh University Press and Ibadan: Heinemann of Nigeria, for the International African Institute.

[6] Berman, A. (1995). Pour une critique des traductions: John Donne. Paris: Gallimard.

[7] Berman, A. (1984). L'Épreuve de l'étranger. Culture et traduction dans l'Allemagne romantique

[8] Collection Les Essais ( ${ }^{\circ} 226$. Paris : Gallimard.

[9] Boufoy-Bastick, B. (2016). Restructurer la politique linguistique de l'UE et défendre la diversité du patrimoine linguistique européen : prévenir la tragédie des communs. Verbum: Vol 7 : Verbum

[10] Bourjea, M. (1986). Traduire ou l'art de voyager. Meta, 31 (3), 231-232.https://doi.org/10.7202/002126ar.

[11] Chaouite, A. (2008). Les maîtres cachés de l'interculturel. Dialogues de cultures : de la traduction Ecarts d'identités.113 (16-20).

[12] Chesterman, A. et Wagner, E. (2002). Can Theory Help Translators? A Dialogue Between the Ivory Tower and the Wordface. Translation Theories Explained Series, Anthony Pym (ed.)Manchester, UK: St Jerome Publishing.

[13] Denti, C. (2017). L'hétérolinguisme ou penser autrement la traduction. Meta, 62 (3), 521-537. https://doi. org/10.7202/1043946ar.

[14] Dongho, J-R. (2015). Analyse systémique de l'environnement socio-psychologique de l'enseignement/apprentissage du français en zone anglophone au Cameroun. L'Enseignement du français en zone anglophone au Cameroun. Sous la direction de Pierre Martial Abessolo. 187-210.Kansas City :Miraclaire Academic Publications.

[15] Dongho, J-R. (2006).The Attitudes of the Anglophone Speech Community towards English-French Bilingualism in Cameroon. A Case Study of Fako Division. Mémoire de M.A. ASTI/Université de Buea. Buea : inédit.

[16] Hersent, J. (2007). Traduire : rencontre ou affrontement entre cultures ? Hermès, La Revue, 49(3), 157167. https://www.cairn.info/revue-hermes-la-revue-2007-3-page-157.htm.

[17] Horguelin, P. (1980). Anthologie de la manière de traduire. Domaine français. Montréal : Linguatech.

[18] KamguemKamgain, H. (2020) Vers une traduction française inclusive deAcross the Mongolo de John NkemngongNkengasong, Mémoire de M.A. ASTI/Université de Buea. Buea : inédit.

[19] Kom, A. (2000).La malédiction francophone. Défis culturels et condition postcoloniale en Afrique. Littératures des Peuples Noirs / African Peoples Literatures ; 2)

[20] Lambert, J. et Gorp, H. (1985). On Describing Translations.The Manipulation of Literature. Theo Hermans (éds).42-53. London/Sydney: CroomHelm. 
[21] Lievois, K. (2018). L'enseignement de la littérature et de la traduction. L. Medjahed, A. Kridech, \& F. Benramdane (éds.), Enseignement-apprentissage de la littérature: base de données, onomastique et comparatisme littéraires. 61-76. Oran: Centre de Recherche en Anthropologie Sociale et Culturelle (CRASC).

[22] Meli, J. (2011). Traduction littéraire au Cameroun: État des lieux et perspectives.Mémoire de M.A. Université de Buea : inédit.

[23] Mopoho, R. (2001). Statut de l'interprète dans l'administration coloniale en Afrique francophone. Meta 46 (3)615-626.

[24] Muna, A. (1991). Lettre à mon frère francophone. Génération, hors-série, (1)25-31 .

[25] NdeffoTene, A. (2015) Promotion du français en zone anglophone : rôle de la traduction. L'Enseignement du français en zone anglophone au Cameroun. Sous la direction de Pierre Martial Abessolo. 255270.Kansas City:MiraclaireAcademic Publications.

[26] Ngoubolong, G. (2020) Traduction et écriture de la résistance dans The Widow'sMight de John NkemngongNkengasong, Mémoire de M.A. Université de Buea : inédit.

[27] Nkemngong, N. J. (2006).The Widow's Might. Yaounde:Editions CLE

[28] Nkemngong, N. J. (2014). Across the Mongolo. Buea: ANUCAM Educational Books plc.

[29] Nkwi, N.W. (2004). The Anglophone Problem. Victor Julius Ngoh (éd.) Cameroon: from a Federal to a Unitary State 1961 - 1972. A CriticalStudy. Limbe : Design House.

[30] Sardin, J. (2007). De la note du traducteur comme commentaire : entre texte, paratexte et prétexte.Palimpsestes (20), 121-136.https://journals.openedition.org/palimpsestes/99.

[31] Sevry J. (1998). Une fidélité impossible : Traduire une œuvre africaine anglophone. Palimpsestes (11)135149, Traduire la culture.

[32] Seleskovitch, D. et Lederer, M. (2014). Interpréter pour traduire. Paris : Éditions Les Belles Lettres. $5^{\mathrm{e}}$ édition revue et corrigée.

[33] Tah, P. (2012). Immortal Seed. Limbe: Pressbook Publishers.

[34] Teinkeu, D. G. (2018).Traduisibilité de l'hybridité de ImmortalSeed de TahProtus. Mémoire de Master, ASTI, Université de Buea : inédit.

[35] Thiele, M. (1967) None but Nightingale. An Introduction to Chinese Literature. Tutland/Tokyo: Cherles E. Tuttle.

[36] Venuti, L. éd. (1992). Rethinking Translation: Discourse, Subjectivity, Ideology.Cornwall: T.J. Press.

[37] Wuilmart, F. (2007). Le péché de « nivellement » dans la traduction littéraire. Meta, 52(3), 391-400. https://doi.org/10.7202/016726ar.

Citation: Jean-Richard DONGHO. "Traduction Et Bilinguisme: Traduire L'altérité À L'heure Du Bilinguisme Et Du Multiculturalisme Au Cameroun” International Journal of Humanities Social Sciences and Education (IJHSSE), vol 8, no. 8, 2021, pp. 176-184. doi: https://doi.org/10.20431/2349-0381.0808017.

Copyright: (c) 2021 Authors. This is an open-access article distributed under the terms of the Creative Commons Attribution License, which permits unrestricted use, distribution, and reproduction in any medium, provided the original author and source are credited. 